'Escuela de Kinesiología, Facultad de Salud, Universidad Santo Tomás. Chile. ${ }^{2}$ Universidad Tecnológica de Chile INACAP, Chile.

${ }^{3}$ Instituto de Actividad Física y Salud, Universidad Autónoma de Chile, Chile.

4Pedagogía en Educación Física, Facultad de Educación, Universidad Autónoma de Chile. Temuco, Chile.

${ }^{5}$ Programa de Doctorado en Ciencias de la Actividad Física,

Facultad de Ciencias de la Educación, Universidad Católica del Maule. Talca, Chile.

${ }^{6} \mathrm{BHF}$ Glasgow Cardiovascular Research Centre, Institute of Cardiovascular and Medical Sciences, University of Glasgow, Glasgow, United Kingdom ${ }^{7}$ Centro de Investigación en Fisiología del Ejercicio - CIFE, Universidad Mayor. Santiago, Chile.

${ }^{a}$ Kinesiólogo.

bProfesor de Educación Física. 'Estudiante pre grado Escuela de Kinesiología, Facultad de Salud, Universidad Santo Tomás. Chile. ${ }^{\mathrm{d} N u t r i c i o n i s t a .}$ eMsC. ${ }^{\mathrm{f}} \mathrm{PhD}$.

Conflicto de interés: Los autores no declaran conflictos de interés. Trabajo no recibió finaciamiento.

Recibido el 15 de abril de 2018, aceptado el 19 julio de 2018.

Correspondencia a: Yeny Concha Cisternas yenyf.concha@gmail.com

\section{Factores de riesgo asociados a bajo nivel de actividad física y exceso de peso corporal en estudiantes universitarios}

\author{
YENY CONCHA-CISTERNAS ${ }^{1,2, \mathrm{a}, \mathrm{e}}$, EDUARDO GUZMÁN-MUÑOZ ${ }^{1, \mathrm{a}, \mathrm{e}}$, \\ PABLO VALDÉS-BADILLA ${ }^{3,4,5, b, e}$, CARLOS LIRA-CEA ${ }^{1, \mathrm{c}}$, \\ FANNY PETERMANN ${ }^{6, \mathrm{~d}, \mathrm{e}}$, CARLOS CELIS-MORALES $^{6,7, \mathrm{~b}, \mathrm{f}}$
}

\section{Levels of physical activity and excess body weight in university students}

Background: University students are considered a vulnerable group due to their adoption of unhealthy diets and lifestyles. Aim: To determine the main risk factors associated with low level of physical activity and excess body weight in university students. Material and Methods: This cross-sectional study included 358 university students from Talca, (53\% female). Sociodemographic, academic, health, lifestyle, nutritional status, physical activity and sedentary behavior variables were analyzed by logistic regression. Results: Men were more likely to present excess body weight than women (Odds ratio (OR): 2.16 [95\% CI: 1.17-4.01], $p=0.01)$. Similarly, those with low level of physical activity (OR: 2.13 [95\% CI: 1.10-414], $p=0.03$ ), musculoskeletal injuries (OR: 2.43 [95\% CI: 1.09-6.34], $p=0.05$ ) and smokers (OR: 2.09 [95\% CI: 1.05-4.14], $p=0.04$ ) were more likely to have excess body weight. Individuals with excess body weight (OR: 2.94 [95\% CI: 1.31-6.61], $p<0.01$ ), high sedentary behaviors (OR: 2.01 [95\% CI: 1.04-4.55], $p=0.04)$, those who spent more than 7 hours per week studying (OR: 2.11 [95\% CI: 1.06-4.66], $p=0.04$ ), and those with presence of musculoskeletal injuries (OR: 1.49 [95\% CI: 1.06- 3.66], $p=0.04$ ) were more likely to be physically inactive. In addition, men were $73 \%$ less likely to have low physical activity levels compared to women (OR: 0.27 [95\% CI: 0.11-0.56], $p<0.01$ ). Conclusions: University students have several modifiable risk factors associated with low levels of physical activity and excess weight. Healthy lifestyles promotion should modify these risk behaviors.

(Rev Med Chile 2018; 146: 840-849)

Key words: Adolescent; Students; Exercise; Obesity; Risk Factors.
E l exceso de peso corporal (sobrepeso y obesidad) se ha convertido en una epidemia mundial a lo largo del ciclo vital, transformándose en un gran desafío para la salud pública por su alta morbilidad y mortalidad ${ }^{1}$. Proyecciones señalan que $20 \%$ de toda la población adulta del mundo será obesa para el año 2030². En Chile, los primeros resultados entregados por la Encuesta Nacional de Salud 2016-2017 muestran una prevalencia de $27,6 \%$ de sobrepeso y $13,2 \%$ de obesidad en jóvenes de 15 a 19 años, mientras que en adultos de 20 a 29 años, estas cifras ascienden a $35,8 \%$ y $24,6 \%$, respectivamente ${ }^{3}$. Datos que sitúan a Chile en la primera posición del ranking de obesidad en Latinoamérica ${ }^{4}$.

Si bien el exceso de peso corporal es un problema multifactorial, que puede originarse por causas ambientales, genéticas y socioeconómicas, la evidencia apunta a la inactividad física y la adopción de estilos de vida poco saludables como 
sus principales causantes ${ }^{5,6}$. En este contexto, estudios sobre población chilena, desarrollados en estudiantes universitarios, han reportado un alto número de conductas y factores de riesgo poco saludables, entre los cuales se identifican un alto número de horas sentados, consumo de alcohol y alimentos procesados (con un alto contenido de sal, grasas saturadas y azúcares simples), no respetar los tiempo de comida como el desayuno, hábito tabáquico, insuficientes horas de sueño, exceso de peso corporal e inactividad física, estando estos dos últimas directamente relacionadas con el desarrollo de enfermedades cardiovasculares ${ }^{7-10}$.

Actualmente, $31,1 \%$ de la población adulta a nivel mundial no cumple las recomendaciones de actividad física (AF) ${ }^{11}$ (150 min de AF moderada o 75 min vigorosos semanales) entregados por el Colegio Americano de Medicina del Deporte ${ }^{12}$, y en Chile, 27,1\% de la población joven reportó ser físicamente inactiva ${ }^{6,13,14}$. Estos datos han generado preocupación en las autoridades, más aun cuando se ha señalado que la inactividad física es responsable de la aparición de enfermedades cardiovasculares y algunos tipos de cáncer ${ }^{15}$. Además, estimaciones la posicionan como la cuarta causa de muerte a nivel mundial ${ }^{16}$.

Considerando que la AF y el exceso de peso son importantes factores de riesgo para el desarrollo de enfermedades cardiovasculares, y que estudios señalan que la prevalencia de inactividad física aumenta drásticamente durante la transición entre la adolescencia y la edad adulta ${ }^{17}$, es fundamental identificar los posibles factores de riesgo asociados con el aumento de esta prevalencia. En consecuencia, el objetivo de este estudio fue determinar los principales factores de riesgo asociados a un bajo nivel de AF y exceso de peso corporal en estudiantes universitarios.

\section{Materiales y Métodos}

\section{Diseño y sujetos de estudio}

Estudio descriptivo de corte transversal, desarrollado entre los meses de abril y mayo del año 2017. Los participantes fueron obtenidos a través de un muestreo no probabilístico por cuotas. Se realizó el cálculo del tamaño muestral considerando una población de 962 estudiantes de la Facultad de Salud de la Universidad Santo Tomás, sede Talca, Chile, un margen de error de $5 \%$ y un nivel de confianza de 95\%. Esto arrojó un tamaño muestral recomendado de 275 estudiantes. Finalmente, la muestra quedó constituida por 358 estudiantes, distribuidos en hombres $(\mathrm{n}=168)$ y mujeres $(\mathrm{n}=190)$.

Los criterios de inclusión utilizados fueron: a) contar con matrícula vigente durante el período en que fueron obtenidos los datos; b) tener $\geq 18$ años de edad; c) aceptar participar voluntariamente del estudio; d) firmar un consentimiento informado.

Fueron excluidos los estudiantes que al momento de la recolección de datos: a) tenían licencia médica; b) se encontraban realizando su práctica profesional; c) estuviesen embarazadas; d) presentaran contraindicaciones permanentes o temporales para realizar AF.

\section{Proceso de registro}

Durante dos semanas se realizó una invitación a participar del estudio. Los voluntarios que aceptaron ser parte de la investigación firmaron un consentimiento informado autorizando el uso de la información con fines de investigación científica. La confidencialidad de sus antecedentes, metodología y objetivo del estudio fueron explicados previo al registro de datos.

\section{Variables en estudio}

Las variables estudiadas fueron: antecedentes sociodemográficos, estado nutricional, antecedentes académicos, antecedentes de salud, estilos de vida, nivel de AF y conducta sedentaria. Los antecedentes sociodemográficos consideraron edad, género y procedencia geográfica (rural o urbana). Para medir el peso corporal se utilizó una balanza electrónica portátil (Seca 769, Alemania; precisión $0,1 \mathrm{~kg}$ ) y la estatura se midió en posición bípeda utilizando un estadiómetro portátil (Seca 217, Alemania; precisión $0,1 \mathrm{~cm}$ ). Ambas mediciones fueron realizadas antes de entregar las encuestas por un profesional capacitado pertenenciente a la Facultad de Salud de la universidad. El estado nutricional se determinó a través del índice de masa corporal (IMC) según los criterios de la Organización Mundial de la Salud (OMS), que señala dividir el peso por la estatura al cuadrado $\left(\mathrm{kg} / \mathrm{m}^{2}\right)^{14}$. A partir de esto, se dicotomizó en normopeso $<24,9 \mathrm{~kg} / \mathrm{m}^{2}$ y exceso de peso $>25,0 \mathrm{~kg} /$ $\mathrm{m}^{2}$. Las variables sobre antecedentes académicos incluyeron el número de horas dedicadas a estudiar en la semana $(\leq 7 \mathrm{y}>7 \mathrm{~h})$, horas inscritas el primer semestre del año académico $2017(<500$ y 
$\geq 500 \mathrm{~h}$ ) y años en la universidad ( $\leq 2 \mathrm{y}>2$ años). Se categorizó la característica años de universidad en $\leq 2 \mathrm{y}>2$, ya que estudios revelan que durante los dos primeros años de universidad ocurren grandes cambios en las conductas saludables de los estudiantes ${ }^{18}$, mientras que el número de horas inscritas en el semestre y horas dedicadas a estudiar, se obtuvieron del promedio reportado por los participantes del estudio. La presencia de enfermedades como diabetes mellitus tipo 2, hipertensión arterial, asma, lesiones músculo-esqueléticas y otros, fueron consultados dentro de la variable antecedentes de salud. Para los datos asociados a estilos de vida, como consumo de alcohol, comida chatarra y hábito tabáquico, se utilizó la clasificación señalada en la Encuesta Nacional de Salud 2009-2010 ${ }^{14}$, para después categorizar a los participantes en los dominios "si consume" o "no consume". Mientas que para el consumo de frutas y verduras se consideró un promedio reportado en investigaciones similares, creándose la variable categórica con puntos de corte $\geq 5$ veces por semana $y<5$ veces por semana ${ }^{19}$. Todas las variables fueron obtenidas mediante autorreporte a través de cuestionarios confeccionados para el estudio.

\section{Clasificación de actividad física}

El nivel de AF se midió a través del Cuestionario Internacional de Actividad Física (IPAQ) versión $\operatorname{corta}^{20}$. El indicador de AF total fue expresado de manera continua en METs (Metabolic-energy-equivalents) y de manera categórica, clasificando el nivel de AF en bajo y moderado/ alto $^{21}$. Se estimó como punto de corte para bajo nivel de AF un gasto energético < $600 \mathrm{METs} / \mathrm{min} /$ semana, $y>600 \mathrm{MET} / \mathrm{min} /$ semana para moderado/alto ${ }^{21,22}$. Se consideró conducta sedentaria cuando los estudiantes destinaban un tiempo $\geq 4 \mathrm{~h}$ al desarrollo de actividades sedentes al día (ej. tiempo sentado frente al computador o TV, o desplazamiento en auto, bus, entre otras), como ha sido señalado en otros estudios en población chilena $^{23,24}$.

\section{Análisis de datos}

Los datos fueron analizados con el software estadístico SPSS 23.0 (SPSS 23.0 para Windows, SPSS Inc., IL, USA). Se utilizaron tablas de contingencia para asociar el estado nutricional y nivel de AF con las distintas variables de interés a través de la prueba de $\chi^{2}$.
Los factores de riesgo asociados a exceso de peso corporal y a bajos niveles de actividad física se identificaron mediante análisis de regresión logística. Estos análisis para exceso de peso fueron ajustados por género, edad, procedencia geográfica y nivel de AF, mientras que los relacionados a bajos niveles de actividad física fueron ajustados por género, edad, procedencia geográfica y estado nutricional. Los datos para estos análisis fueron presentados como Odds ratio (OR) y sus respectivos intervalos de confianza de $95 \%$ [95\% CI]. El nivel de significancia fue definido como $\mathrm{p}<0,05$.

\section{Resultados}

La Tabla 1 muestra las características generales de los participantes según estado nutricional. La mayor prevalencia de exceso de peso corporal se observó en hombres y en estudiantes con bajo nivel de AF, presencia de lesión musculo-esquelética y hábito de fumar. En la Tabla 2 se observa las características de los participantes según nivel de AF. La mayor prevalencia de bajos niveles de AF fue en mujeres y en estudiantes con exceso de peso corporal, conducta sedentaria, más de $7 \mathrm{~h}$ dedicadas a estudiar por semana y presencia de lesión músculo-esquelética.

Los factores asociados con exceso de peso corporal son presentados en la Figura 1. Los resultados muestran que los hombres (OR: 2,16 [95\% CI: $1,17-4,01], \mathrm{p}=0,014)$ tienen 2,16 veces más probabilidad que las mujeres de presentar dicha condición. Además, el bajo nivel de AF (OR: 2,13 [95\% CI: 1,10-4,14], $\mathrm{p}=0,026)$, la presencia de lesiones músculo-esqueléticas (OR: 2,43 [95\% CI: $1,09-6,34], \mathrm{p}=0,049)$ y el hábito tabáquico (OR: 2,09 [95\% CI: 1,05-4,14], $\mathrm{p}=0,036$ ) son factores que se asocian significativamente al exceso de peso corporal.

En la Figura 2, los resultados señalan que los hombres tienen $73 \%$ menor probabilidad de presentar bajos niveles de AF en comparación a las mujeres (OR: 0,27 [95\% CI: 0,11-0,56], $\mathrm{p}=0,001)$. Además, el exceso de peso corporal (OR: 2,94 [95\% CI: 1,31-6,61], $\mathrm{p}=0,009$ ), una conducta altamente sedentaria (OR: 2,01 [95\% CI: 1,04-4,55], $\mathrm{p}=0,040)$, dedicar al estudio más de $7 \mathrm{~h}$ a la semana (OR: 2,11 [95\% CI: 1,06-4,66], $\mathrm{p}=0,043)$ y presentar lesiones músculo-esqueléticas (OR:1,49 [95\% CI: 1,06-3,66], $\mathrm{p}=0,043$ ) son factores asociados a presentar bajos niveles de AF. 
Tabla 1. Características de la muestra según estado nutricional

\begin{tabular}{|c|c|c|c|}
\hline & \multicolumn{2}{|c|}{ Estado nutricional } & \multirow[b]{2}{*}{ Valor $\mathbf{p}$} \\
\hline & $\begin{array}{c}\text { Normopeso } \\
(n=218)\end{array}$ & $\begin{array}{l}\text { Exceso de peso } \\
\qquad(n=140)\end{array}$ & \\
\hline \multicolumn{4}{|l|}{ Género } \\
\hline Mujeres & 60,6 & 41,4 & \multirow{2}{*}{0,009} \\
\hline Hombres & 39,4 & 58,6 & \\
\hline \multicolumn{4}{|l|}{ Edad } \\
\hline$<21$ años & 50,5 & 44,3 & \multirow{2}{*}{0,257} \\
\hline$\geq 21$ años & 49,5 & 55,7 & \\
\hline \multicolumn{4}{|l|}{ Procedencia geográfica } \\
\hline Rural & 17,4 & 22,8 & \multirow{2}{*}{0,241} \\
\hline Urbano & 82,6 & 77,2 & \\
\hline \multicolumn{4}{|l|}{ Nivel de actividad física } \\
\hline Moderado/alto & 57,8 & 41,4 & \multirow{2}{*}{0,032} \\
\hline Bajo & 42,2 & 58,6 & \\
\hline \multicolumn{4}{|l|}{ Conducta sedentaria } \\
\hline No & 32,1 & 37,1 & \multirow{2}{*}{0,297} \\
\hline Sí & 67,9 & 62,9 & \\
\hline \multicolumn{4}{|l|}{ Antecedentes académicos (\%) } \\
\hline$>2$ años en la universidad & 44,1 & 37,2 & 0,224 \\
\hline$\geq 500 \mathrm{~h}$ inscritas al semestre & 56,9 & 57,2 & 0,548 \\
\hline$>7 \mathrm{~h}$ dedicadas al estudio por semana & 56,0 & 67,2 & 0,090 \\
\hline \multicolumn{4}{|l|}{ Presencia de enfermedades } \\
\hline Diabetes mellitus tipo 2 & 0,0 & 1,5 & 0,391 \\
\hline Hipertensión arterial & 1,8 & 0,0 & 0,396 \\
\hline Asma & 6,4 & 5,7 & 0,558 \\
\hline Lesión músculo-esquelética & 8,3 & 18,6 & 0,037 \\
\hline Otras enfermedades & 11,1 & 10,0 & 0,520 \\
\hline \multicolumn{4}{|l|}{ Hábitos } \\
\hline Fuma & 23,9 & 37,1 & 0,041 \\
\hline Consume alcohol & 48,6 & 47,2 & 0,484 \\
\hline Consume comida chatarra & 48,6 & 40,0 & 0,164 \\
\hline Consume frutas $\geq 5$ veces por semana & 50,5 & 50,0 & 0,537 \\
\hline Consume verduras $\geq 5$ veces por semana & 31,2 & 38,6 & 0,196 \\
\hline
\end{tabular}

Valores expresados en frecuencias relativas (\%). Valor p: prueba de asociación $\chi^{2}$. 
Tabla 2. Características de la muestra según nivel de actividad física

\begin{tabular}{|c|c|c|c|}
\hline & \multicolumn{2}{|c|}{ Nivel de actividad física } & \multirow[b]{2}{*}{ Valor $\mathbf{p}$} \\
\hline & $\begin{array}{l}\text { Moderado / Alto } \\
(n=192)\end{array}$ & $\begin{array}{c}\text { Bajo } \\
(n=166)\end{array}$ & \\
\hline \multicolumn{4}{|l|}{ Género } \\
\hline Mujeres & 40,6 & 67,5 & \multirow{2}{*}{$<0,001$} \\
\hline Hombres & 59,4 & 32,5 & \\
\hline \multicolumn{4}{|l|}{ Edad } \\
\hline$<21$ años & 48,9 & 47,0 & \multirow{2}{*}{0,455} \\
\hline$\geq 21$ años & 51,1 & 53,0 & \\
\hline \multicolumn{4}{|l|}{ Procedencia geográfica } \\
\hline Rural & 22,9 & 15,7 & \multirow{2}{*}{0,151} \\
\hline Urbano & 77,1 & 84,3 & \\
\hline \multicolumn{4}{|l|}{ Estado nutricional } \\
\hline Normopeso & 70,6 & 44,6 & \multirow{2}{*}{0,014} \\
\hline Exceso de peso corporal & 29,4 & 55,4 & \\
\hline \multicolumn{4}{|l|}{ Conducta sedentaria } \\
\hline No & 40,6 & 26,5 & \multirow{2}{*}{0,033} \\
\hline Sí & 59,4 & 73,5 & \\
\hline \multicolumn{4}{|l|}{ Antecedentes académicos (\%) } \\
\hline$>2$ años en la universidad & 57,3 & 60,2 & 0,403 \\
\hline$\geq 500 \mathrm{~h}$ inscritas al semestre & 42,7 & 43,4 & 0,524 \\
\hline$>7 \mathrm{~h}$ dedicadas al estudio por semana & 45,8 & 32,5 & 0,048 \\
\hline \multicolumn{4}{|l|}{ Presencia de enfermedades } \\
\hline Diabetes mellitus tipo 2 & 1,0 & 0,0 & 0,536 \\
\hline Hipertensión arterial & 2,1 & 1,2 & 0,286 \\
\hline Asma & 6,3 & 6,2 & 0,600 \\
\hline Lesión músculo-esquelética & 8,8 & 7,2 & 0,031 \\
\hline Otras enfermedades & 10,4 & 10,8 & 0,558 \\
\hline \multicolumn{4}{|l|}{ Hábitos } \\
\hline Fuma & 31,3 & 26,5 & 0,298 \\
\hline Consume alcohol & 53,1 & 46,8 & 0,094 \\
\hline Consume comida chatarra & 42,7 & 48,2 & 0,055 \\
\hline Consume frutas $\geq 5$ veces por semana & 48,9 & 51,8 & 0,409 \\
\hline Consume verduras $\geq 5$ veces por semana & 30,2 & 23,8 & 0,554 \\
\hline
\end{tabular}

Valores expresados en frecuencias relativas (\%). Valor p: prueba de asociación $\chi^{2}$. 


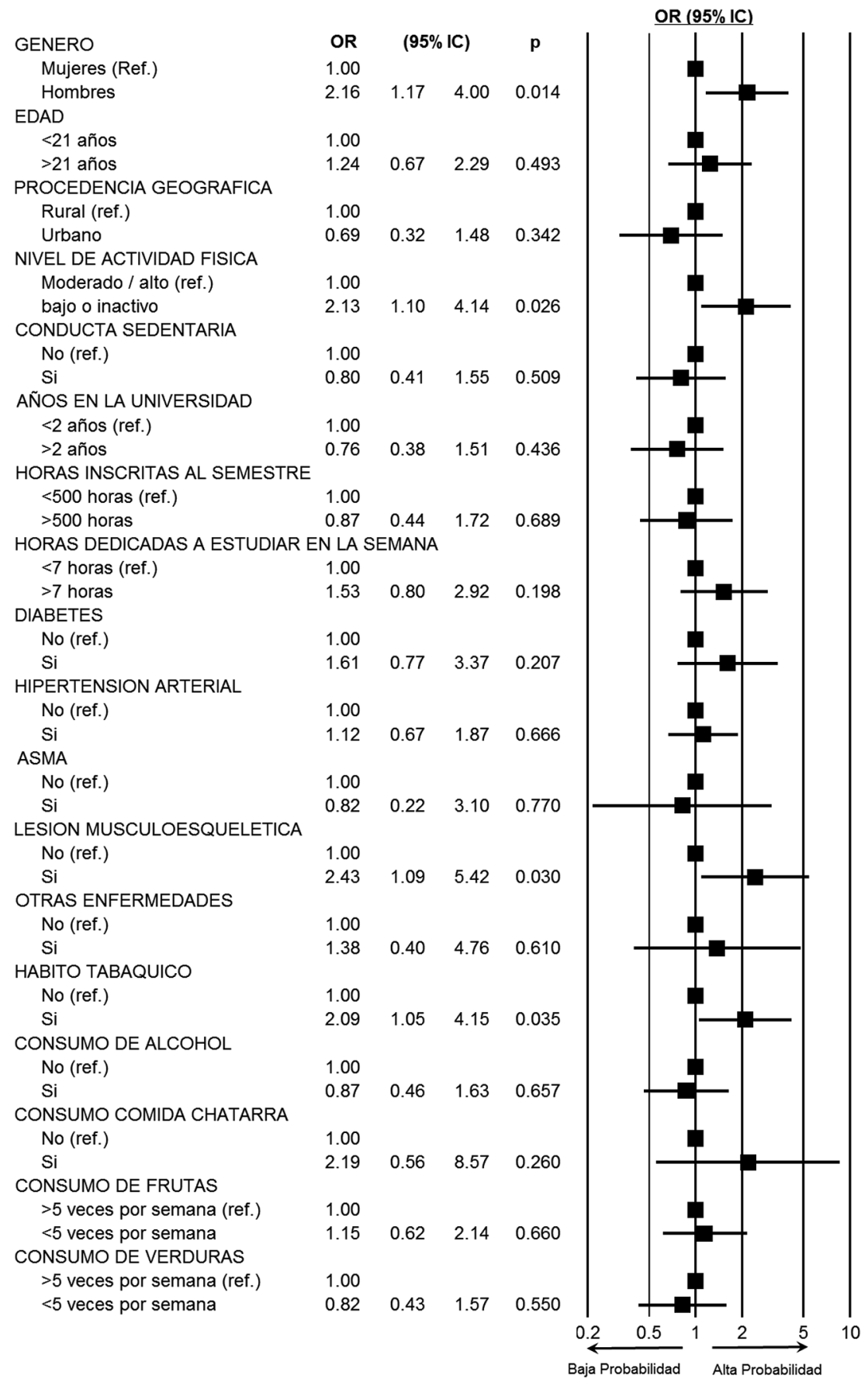

Figura 1. Factores asociados a exceso de peso corporal en universitarios. Datos presentados como odds ratio y sus respectivos intervalos de confianza de 95\%. Todos los análisis fueron ajustados por sexo, edad, procedencia geográfica y nivel de AF. El grupo de referencia utilizado para cada variable se señala con (ref.). Un valor de OR $>1$ indica una alta probabilidad de tener exceso de peso, por ende, un valor de $\mathrm{OR}<1$ indica una baja probabilidad de tener exceso de peso.

Ref.: Referencia; OR: Odds ratio; IC: intervalos de confianza. Valor p: prueba de regresión logística. 


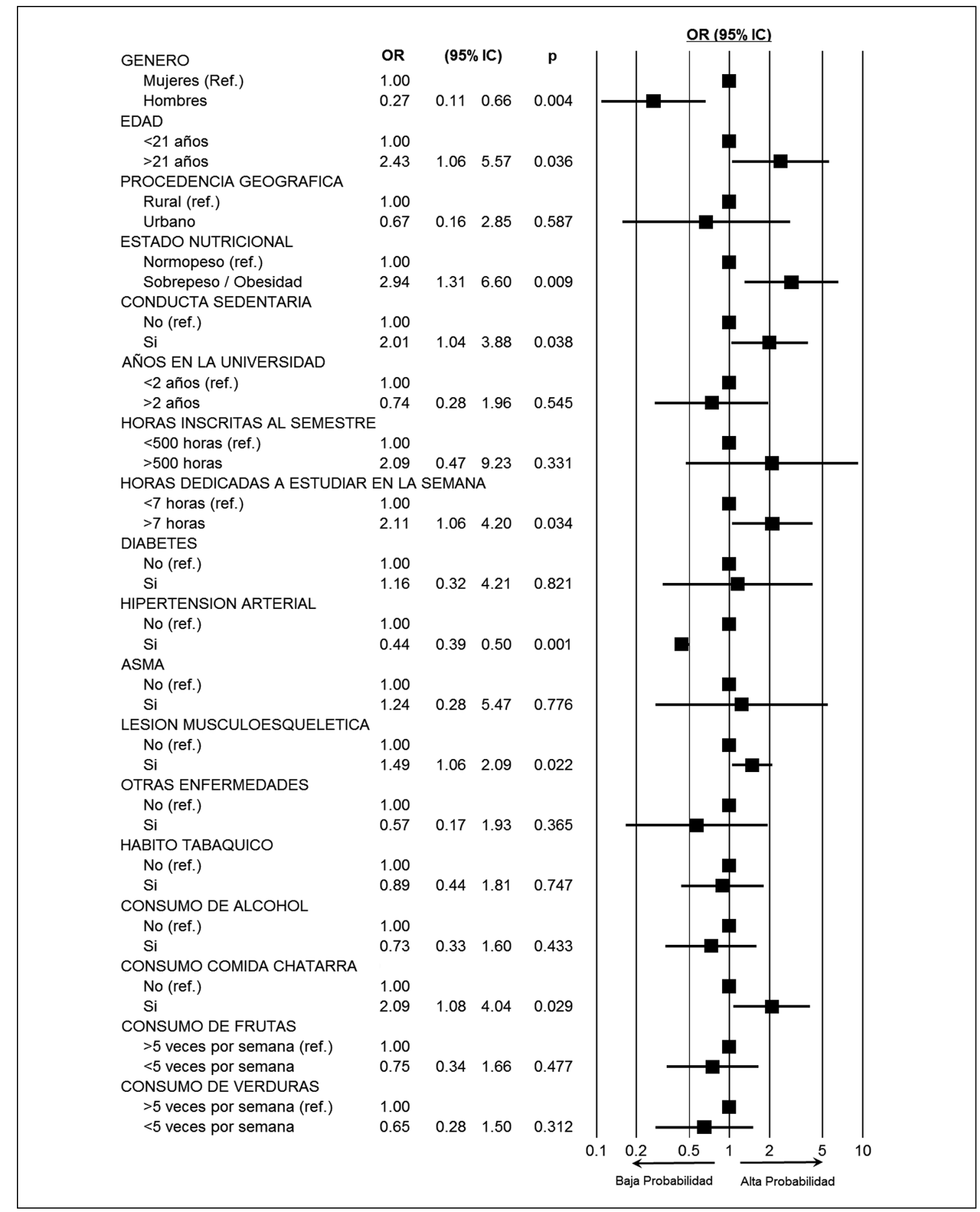

Figura 2. Factores asociados a bajo nivel de AF en universitarios. Datos presentados como odds ratio y sus respectivos intervalos de confianza de $95 \%$. Todos los análisis fueron ajustados por sexo, edad, procedencia geográfica y estado nutricional. El grupo de referencia utilizado para cada variable se señala con (ref.). Un valor de OR mayor a 1 indica una alta probabilidad de realizar bajos niveles de actividad física, por ende, un valor de $\mathrm{OR}<1$ indica una baja probabilidad de realizar bajos niveles de actividad física. Ref.: Referencia; OR: Odds ratio; IC: intervalos de confianza. Valor p: prueba de regresión logística. 


\section{Discusión}

Los principales resultados de este estudio señalan que pertenecer al género masculino, tener hábito tabáquico, antecedentes de lesión músculo-esquelética y bajo nivel de AF, se asocian a mayor probabilidad de presentar exceso de peso. Mientras que ser mayor de 21 años, mantener una conducta sedentaria, ser obeso o tener sobrepeso, presentar antecedentes de lesión músculo-esquelética, consumir comida altamente chatarra y destinar una mayor cantidad de horas de estudio se asocia con bajo nivel de AF. Estos resultados podrían ser de utilidad para la implementación de programas de promoción de estilos de vida saludable en la población universitaria de Chile, la cual, como se ha expuesto en estudios anteriores, presenta una alta prevalencia de conductas no saludables durante su formación universitaria.

Estos hallazgos concuerdan con los reportados por otras investigaciones, las cuales revelan que el hábito tabáquico y la inactividad física contribuyen con el desarrollo de sobrepeso y obesidad ${ }^{1,25}$. Del mismo modo, un estudio realizado en universitarios chilenos identificó que hombres presentaban un mayor exceso de peso corporal (>25 kg) $\mathrm{m}^{2}$ ) y mayor consumo de tabaco, transformando de este modo al género en un posible factor de riesgo. Este hallazgo es atribuido a que los varones consumirían mayor cantidad de bebidas azucaradas, alcohol y comida chatarra ${ }^{26}$. En contraste, Petermann y cols. ${ }^{21}$ evidenciaron que los factores de riesgo asociados a ser obeso difieren por género, señalando a las mujeres como el grupo de mayor riesgo (OR: 1,$41 ; \mathrm{p}<0,0001$ ). La discrepancia en los resultados obtenidos por nuestro estudio podría atribuirse a las diferencias entre los grupos de edad elegidos, lo que indicaría la aparición de conductas relacionadas con el avance de la edad.

En Chile, $45,5 \%$ de los universitarios declaran mantener un hábito diario u ocasional de tabaco ${ }^{26,27}$. Nuestra investigación evidenció que el hábito tabáquico podría considerarse un factor de riesgo para el desarrollo de sobrepeso y obesidad. Estos resultados concuerdan con estudios nacionales previos, los que señalan la existencia de asociación entre exceso de peso con el consumo de cigarrillos y otros hábitos poco saludables ${ }^{7,28}$. En este contexto y considerando la relación existente entre hábito tabáquico, el desarrollo de enfermedades crónicas y algunos tipos de cáncer, los universitarios son una población altamente vulnerable, que requiere acciones de prevención inmediatas, con el objetivo de evitar deterioros en la salud en el corto a mediano plazo.

Por otra parte, revisiones sistemáticas y estudios realizados en población chilena señalan que mantener una conducta sedentaria y un bajo nivel de AF se asocian a una mayor probabilidad de ser obeso, principalmente en hombres ${ }^{29-31}$, coincidiendo estos hallazgos con los resultados observados en nuestra investigación. A pesar de las diferentes estrategias desarrolladas para controlar la alta prevalencia de sobrepeso y obesidad en diferentes rangos etarios, aún es necesario generar mayores instancias de promoción de la práctica regular de AF y hábitos alimentarios saludable en la población chilena.

En cuanto a los factores asociados con bajo nivel de AF, la literatura ha expuesto similares resultados a los reportados en nuestra investigación ${ }^{23,24,32}$. En la actualidad, el consumo de comida chatarra es considerado un factor importante para el desarrollo de exceso de peso, mientras que tener exceso de peso se asocia con mayor riesgo de no cumplir las recomendaciones mínimas de $\mathrm{AF}^{23,32,33}$. Esta secuencia de hábitos poco saludables favorece el desarrollo de comorbilidades como diabetes mellitus tipo 2, hipertensión arterial, síndrome metabólico, entre otras ${ }^{6,23,29}$. En concordancia con lo expuesto, nuestro estudio indica que la ingesta de comida chatarra se asocia también a bajo nivel de $\mathrm{AF}$, respaldando la necesidad de educar en temáticas saludables.

Si bien diversos estudios han investigado la asociación entre la práctica regular de AF con diferentes factores (p.e. sociodemográficos, estilos de vida, antecedentes de salud, entre otros) muy pocos han incorporado los antecedentes académicos y la relación que estos pudiesen tener con los niveles de AF en población universitaria. Esta investigación revela que los estudiantes que destinan más horas de estudio a la semana, muestran bajos niveles de AF. Esto podría deberse principalmente al tiempo que demandan las obligaciones académicas, dejando escaso tiempo para la práctica de AF. Además, los universitarios mantienen, mayormente, una conducta sedentaria, ya que deben permanecer sentados durante varias horas del día, lo que ha sido considerado como un factor de riesgo para el deterioro de la salud ${ }^{34}$. Estos hallazgos dejan al descubierto la necesidad de implementar 
programas de $\mathrm{AF}$ en la población universitaria, que permitan a los estudiantes ocupar sus tiempos libres de forma saludable dentro de la universidad.

Dentro de las limitaciones de este estudio se encuentra la selección de los participantes a partir de un muestreo no probabilístico, lo cual puede restringir la representatividad externa del estudio. Por otro lado, algunos datos fueron obtenidos mediante autorreporte de AF y uso de encuestas, lo que podría ocasionar un sesgo por la sobre estimación de estudiantes con estilos de vida no saludable.

En conclusión, los estudiantes universitarios que participaron del estudio presentan factores de riesgo asociados con bajos niveles de $\mathrm{AF}$ y exceso de peso, factores que en su mayoría son modificables, excepto el género y la edad. Esto sugiere la necesidad de promover acciones orientadas a contrarrestar los factores de riesgo modificables y promover estilos de vida saludables en la educación superior.

\section{Referencias}

1. Zhang Y, Liu J, Yao J, Ji G, Qian L, Wang J, et al. Obesity: Pathophysiology and Intervention. Nutrients 2014; 6 (11): 51-3.

2. Kelly T, Yang W, Chen CS, Reynolds K, He J. Global burden of obesity in 2005 and projections to 2030. Int J Obes 2008; 32 (9): 1431-7.

3. Ministerio de Salud. Encuesta Naciona de Salud 20162017: primeros resultados. 2017. http://www.minsal.cl/ wp-content/uploads/2017/11/ENS-2016-17_PRIMEROS-RESULTADOS.pdf

4. OMS/OPS. Panorama de la Seguridad Alimentaria y Nutricional en América Latina y el Caribe. Organización de las Naciones Unidas para la Alimentación y la Agricultura, Organización Panamericana de la Salud. Santiago, Chile 2016. http://www.fao.org/3/a-i6747s.pdf

5. von Deneen KM, Liu YJ. Obesity as an addiction: Why do the obese eat more? Maturitas 2011; 68 (4): 342-5.

6. Celis-Morales C, Salas C, Álvarez C, Aguilar Farías N, Ramírez Campillos R, Leppe J, et al. Un mayor nivel de actividad física se asocia a una menor prevalencia de factores de riesgo cardiovascular en Chile: resultados de la Encuesta Nacional de Salud 2009-2010. Rev Med Chile 2015; 143: 1435-43.

7. Ratner GR, Hernández JP, Martel AJ, Atalah SE. Calidad de la alimentación y estado nutricional en estudiantes universitarios de 11 regiones de Chile. Rev Med Chile
2012; 140: 1571-9.

8. Rodríguez RF, Palma LX, Romo BÁ, Escobar BD, Aragú GB, Espinoza OL, et al. Hábitos alimentarios, actividad física y nivel socioeconómico en estudiantes universitarios de Chile. Nutr Hosp 2013; 28: 447-55.

9. Cervera Burriel F, Serrano Urrea R, Vico García C, Milla Tobarra M, García Meseguer MJ. Hábitos alimentarios y evaluación nutricional en una población universitaria. Nutr Hosp 2013; 28: 438-46.

10. Celis-Morales C, Salas C, Alduhishy A, Sanzana R, Martínez MA, Leiva A, et al. Socio-demographic patterns of physical activity and sedentary behaviour in Chile: results from the National Health Survey 2009-2010. J Public Health 2016; 38 (2): E98-E105.

11. García Hermoso A, Saavedra J, Ramírez Vélez R, Ekelund U, Pozo Cruz B. Reallocating sedentary time to moderate to vigorous physical activity but not to light intensity physical activity is effective to reduce adiposity among youths: a systematic review and meta analysis. Obes Rev 2017; 18 (9): 1088-95.

12. Garber CE, Blissmer B, Deschenes MR, Franklin BA, Lamonte MJ, Lee I-M, et al. American College of Sports Medicine position stand. Quantity and quality of exercise for developing and maintaining cardiorespiratory, musculoskeletal, and neuromotor fitness in apparently healthy adults: guidance for prescribing exercise. Med Sci Sports Exer 2011; 43 (7): 1334-59.

13. Ekelund U DP-CB. Reallocating sedentary time to moderate-to-vigorous physical activity but not to light-intensity physical activity is effective to reduce adiposity among youths: a systematic review and meta-analysis. Obes Rev 2017; 9: 1088-95.

14. Ministerio de Salud (MINSAL). Encuesta Nacional de Salud 2009-2010. Chile: Ministerio de Salud; 2010. http://www.minsal.cl/portal/url/item/bcb03d7bc28b64dfe040010165012d23.pdf.

15. Lee IM, Shiroma EJ, Lobelo F, Puska P, Blair SN, Katzmarzyk PT, et al. Effect of physical inactivity on major non-communicable diseases worldwide: an analysis of burden of disease and life expectancy. Lancet 2012; 380 (9838): 219-29.

16. WHO. Global Helath Risk: mortality and burden of disease attributable to selected major risk. Geneva: World Health Organization,2009. http://www.who.int/ healthinfo/global_burden_disease/GlobalHealthRisks_report_full.pdf

17. van Mechelen W, Twisk JWR, Post GB, Snel J, Kemper HCG. Physical activity of young people: the Amsterdam Longitudinal Growth and Health Study. Med Sci Sports Exer 2000; 32 (9): 1610-6.

18. Deliens T, Deforche B, De Bourdeaudhuij I, Clarys P. 
Changes in weight, body composition and physical fitness after 1.5 years at university. Eur J Clin Nutr 2015; 69 (12): 1318.

19. Olivares CS, Lera ML, Bustos ZN. Etapas del cambio, beneficios y barreras en actividad física y consumo de frutas y verduras en estudiantes universitarios de Santiago de Chile. Rev Chil Nutr 2008; 35: 25-35.

20. Román Viñas B, Ribas Barba L, Ngo J, Serra Majem L. Validity of the international physical activity questionnaire in the Catalan population (Spain). Gat Sanit 2013; 27 (3): 254-7.

21. Serón P, Muñoz S, Lanas F. Nivel de actividad física medida a través del cuestionario internacional de actividad física en población Chilena. Rev Med Chile 2010; 138: 1232-9.

22. Ainsworth BE, Haskell WL, Herrmann SD, Meckes N, Bassett DR, Tudor-Locke C, et al. 2011 Compendium of Physical Activities: A Second Update of Codes and MET Values. Med Sci Sports Exer 2011; 43 (8): 1575-81.

23. Díaz X, Garrido A, Martínez MA, Leiva AM, Álvarez C, Ramírez-Campillo R, et al. Factores asociados a inactividad física en Chile: resultados de la Encuesta Nacional de Salud 2009-2010. Rev Med Chile 2017; 145 (10): 1259-67.

24. Martínez MA, Leiva AM, Petermann F, Garrido A, Díaz $\mathrm{X}$, Alvarez C, et al. Factores asociados a sedentarismo en Chile: evidencia de la encuesta nacional de salud 20092010. Rev Med Chile 2018; 146: 22-31.

25. Villablanca AC, McDonald JM, Rutledge JC. Smoking and cardiovascular disease. Clin Chest Med 2000; 21 (1): 159-72.

26. Durán S, Crovetto M, Espinoza V, Mena F, Oñate G, Fernández $\mathrm{M}$, et al. Caracterización del estado nutricional, hábitos alimentarios y estilos de vida de estudiantes universitarios chilenos: estudio multicéntrico. Rev Med
Chile 2017; 145: 1403-11.

27. Nerín I, Crucelaegui A, Novella P, Ramón y Cajal P, Sobradiel N, Gericó R. Encuesta sobre tabaquismo en estudiantes universitarios en relación con la práctica de ejercicio físico. Arch Bronconeumol 2004; 40: 5-9.

28. González Sandoval CE, Díaz Burke Y, Mendizabal-Ruiz AP, Medina Díaz E, Morales JA. Prevalencia de obesidad y perfil lipídico alterado en jóvenes universitarios. Nutr Hosp 2014; 29: 315-21.

29. Petermann F, Durán E, Labraña AM, Martínez MA, Leiva AM, Garrido-Méndez A, et al. Factores asociados al desarrollo de obesidad en Chile: resultados de la Encuesta Nacional de Salud 2009-2010. Rev Med Chile 2017; 145 (6): 716-22.

30. Nocon M, Hiemann T, Mueller-Riemenschneider F, Thalau F, Roll S, Willich SN. Association of physical activity with all-cause and cardiovascular mortality: a systematic review and meta-analysis. Eur J Cardiovasc Prev Rehabil 2008; 15 (3): 239-46.

31. Cenarruzabeitia JJV, Hernández JAM, Martínez-González MÁ. Beneficios de la actividad física y riesgos del sedentarismo. Med Clin 2003; 121 (17): 665-72.

32. Hernández-Escolar J, Herazo-Beltrán Y, Valero MV. Frecuencia de factores de riesgo asociados a enfermedades cardiovasculares en población universitaria joven. REv Salud Publica 2010; 12: 852-64.

33. Pedrozo W, Rascón MC, Bonneau G, Pianesi MId, Olivera CC, Aragón SJd, et al. Metabolic syndrome and risk factors associated with life style among adolescents in a city in Argentina, 2005. Rev Panam Salud Publ 2008; 24 (3): 149-60.

34. Hallal PC, Andersen LB, Bull FC, Guthold R, Haskell W, Ekelund U, et al. Global physical activity levels: surveillance progress, pitfalls, and prospects. Lancet 2012; 380: (9838): 247-57. 